\title{
Concurrent Ventral Hernia Repair Is Effective in Patients Undergoing Abdominally Based Microsurgical Breast Reconstruction
}

\author{
Casey T. Kraft, MD ${ }^{1}$ Albert H. Chao, MD ${ }^{1}$ \\ ${ }^{1}$ Department of Plastic Surgery, Ohio State University Wexner \\ Medical Center, Columbus, Ohio \\ J Reconstr Microsurg 2020;36:572-576.
}

\begin{abstract}
Address for correspondence Albert H. Chao, MD, Department of Plastic Surgery, Ohio State University Wexner Medical Center, 915 Olentangy River Road, Suite 2100, Columbus, OH 43212 (e-mail: Albert.Chao@osumc.edu).
\end{abstract}

\begin{abstract}
Keywords

Background The abdomen remains the most preferable donor site for autologous breast reconstruction. Many patients in this population will have had prior abdominal surgery, which is the chief risk factor for having a ventral hernia. While prior studies have examined the impact of prior abdominal surgery on breast reconstruction, limited data exist on the management of patients with a preexisting ventral hernia. The objective of this study was to investigate outcomes of performing ventral hernia repair concurrent with abdominally based microsurgical breast reconstruction.

Methods A 5-year retrospective review of patients undergoing abdominally based microsurgical breast reconstruction was performed. The experimental group consisted of patients with a preexisting ventral hernia that was repaired at the time of breast reconstruction, and was compared with a historical cohort of patients without preexisting hernias.

Results There were a total of 18 and 225 patients in the experimental and control groups, respectively. There was a higher incidence of prior abdominal surgery in the experimental group $(p=0.0008)$, but no other differences. Mean follow-up was $20.5 \pm 5.2$ months. There were no instances of recurrent hernia or flap loss in the experimental group. No significant differences were observed between the experimental and control groups in the incidence of donor-site complications ( $27.8 \mathrm{vs.} 20.9 \%$, respectively; $p=0.55$ ), recipient site complications ( 27.8 vs. $24.0 \%$, respectively; $p=0.78$ ), operative time ( $623 \pm 114$ vs. $598 \pm 100$ minutes, respectively; $p=0.80$ ), or length of stay ( $3.4 \pm 0.5$ vs. $3.1 \pm 0.4$ days, respectively; $p=0.98$ ).

- breast reconstruction

- hernia Conclusion Concurrent ventral hernia repair at the time of abdominally based microsurgical breast reconstruction appears to be safe and effective. Larger studies

- hernia repair are needed to further define this relationship.
\end{abstract}

Autologous techniques continue to be a mainstay of options for breast reconstruction. The advantages of autologous breast reconstruction include a more natural result, and avoidance of issues associated with alloplastic reconstruction such as the potential for future surgery. In addition, free tissue transfer continues to play an important role in commonly encountered clinical scenarios, such as breast

received

January 12, 2020

accepted

April 20, 2020

published online

June 15,2020 reconstruction after postmastectomy radiation therapy. Although numerous donor sites have been described, the abdomen remains the most preferable donor site because it allows for simultaneous harvest during mastectomy, involves no patient position changes, results in aesthetically favorable scar placement, and has low donor-site morbidity. ${ }^{1-3}$

Copyright @ 2020 by Thieme Medical Publishers, Inc., 333 Seventh Avenue, New York, NY 10001, USA. Tel: $+1(212) 760-0888$
DOI https://doi.org/ 10.1055/s-0040-1713149. ISSN 0743-684X. 
The incidence of breast cancer peaks among women between the ages of 70 and 74 years, by which time many patients will have previously had abdominal surgery. ${ }^{4}$ Previously published studies have reported the incidence of prior abdominal surgery in patients undergoing microsurgical breast reconstruction to be as high as $51 \% .^{5-7}$ Patients with a history of prior abdominal surgery may have alterations in perfusion, more challenging flap dissection, and disruption of perforators or even the flap pedicle. Although many patients who have had prior abdominal surgery may not develop a ventral hernia, prior abdominal surgery is nevertheless one of the primary risk factors for development of a ventral hernia. Thus, reconstructive surgeons are likely to encounter breast cancer patients presenting for breast reconstruction who have had prior abdominal surgery and an associated incisional hernia.

Prior studies investigating abdominally based breast reconstruction in the setting of prior abdominal surgery have focused on flap perfusion and vascularity in patients without ventral hernias. ${ }^{5,8,9}$ However, the subject of outcomes of patients with preexisting ventral hernias undergoing abdominally based breast reconstruction remains relatively unaddressed in the literature. With respect to the flap, the presence of an underlying hernia may cause harvest to be more difficult, affect perfusion, or prolong operative time. With respect to the donor site, the index breast reconstruction operation would intuitively seem to be the most opportune time for hernia repair. However, hernia repair at the time of breast reconstruction may theoretically place the patient at increased risk of complications such as mesh infection (e.g., due to long operative times and multiple open surgical sites) and hernia recurrence (e.g., due to fascial incision/excision and muscle dissection performed during flap elevation).

The objective of this study was to evaluate outcomes of patients undergoing abdominally based microsurgical breast reconstruction who also underwent concurrent repair of a preexisting ventral hernia. These patients were compared with a historical control group of patients without preexisting hernias. Primary outcome measures were the incidences of donor- and recipient-site complications. Secondary outcome measures included length of stay, operative time, and readmission and reoperation rates. We hypothesized that the presence of a ventral hernia would not impact the ability to successfully perform the breast reconstruction. In addition, we hypothesized that hernia repair at the time of breast reconstruction could be performed without adversely affecting the incidence of donor-site complications including hernia recurrence.

\section{Methods}

Following Institutional Review Board approval, we performed a retrospective review of all patients who underwent abdominally based microsurgical breast reconstruction by six plastic surgeons at our institution between 2014 and 2019. The inclusion criteria for the experimental group were patients who also concurrently underwent repair of a preexisting ventral hernia. The experimental group was compared with a historical control group that consisted of patients who did not have a preexisting ventral hernia. A minimum of 6 months of postoperative follow-up was required for both groups. Due to the relatively larger size of the control group, patients in the control group were restricted to a 1-year period between 2018 and 2019. Patients were excluded from the study if they underwent additional reconstructive surgery procedures (e.g., contralateral symmetry, capsulectomy, implant removal) concurrent with breast reconstruction, which might confound the study outcome measures.

Preoperative characteristics, intraoperative details, and postoperative complications and course were recorded. The experimental and control groups were first compared with respect to their preoperative characteristics and intraoperative details to identify any potential confounding factors. Patients who were actively smoking at the time of their breast reconstruction were classified as "smoking." With respect to timing of reconstruction, patients who underwent bilateral breast reconstruction that involved delayed reconstruction on one side and immediate reconstruction on the contralateral side were defined as having "mixed" timing. The two groups were then compared with respect to their outcomes. The independent variable of the study was whether or not a patient underwent concurrent ventral hernia repair at the time of abdominally based microsurgical breast reconstruction. The primary dependent variables were the incidences of donor- and recipient-site complications. Secondary dependent variables analyzed in the study were length of stay, operative time, and the rates of readmission and reoperation within 30 days.

Frequency data were compared using the Fisher's test or chisquare test, as appropriate. Continuous data were evaluated for normality using the Shapiro-Wilk's test and histogram plot. Nonnormally distributed continuous data are reported as medians with interquartile ranges and were compared using the Mann-Whitney's $U$ test. Normally distributed continuous data are reported as means with standard deviations and were compared using the $t$-test. Continuous data were compared using the Mann-Whitney's $U$ test. All tests were two tailed. The $p$-values $<0.05$ were considered significant.

\section{Results}

A total of 18 patients met inclusion criteria for the experimental group and 225 patients met inclusion criteria for the control group. Preoperative characteristics of the study population are summarized in - Table 1. Patients in the experimental group were more likely to have undergone prior abdominal surgery than patients in the control group (94.4 vs. $54.7 \%$, respectively; $p=0.0008$ ), most commonly, hysterectomy (50.0\%), laparoscopic appendectomy (22.2\%), laparoscopic cholecystectomy (22.2\%), and hernia repair (16.7\%). Eight patients (50\%) had a history of an open abdominal procedure, such as a cholecystectomy, hysterectomy, splenectomy, or exploratory laparotomy. Hernias were identified preoperatively by physical examination. Fourteen patients (77.8\%) had midline ventral hernias and four (22.2\%) patients had lateral ventral hernias. Of the 14 midline ventral hernias, $2(11.1 \%)$ were umbilical hernias. All hernias were 
Table 1 Preoperative characteristics of study population

\begin{tabular}{|l|l|l|l|}
\hline & $\begin{array}{l}\text { Concurrent } \\
\text { VHR }(\boldsymbol{n}=\mathbf{1 8})\end{array}$ & $\begin{array}{l}\text { No VHR } \\
(\boldsymbol{n}=\mathbf{2 2 5})\end{array}$ & $p$-Value \\
\hline Age (y) & $53.3 \pm 10.1$ & $56.1 \pm 12.0$ & 0.94 \\
\hline BMI & $30.8 \pm 5.7$ & $32.1 \pm 6.0$ & 0.90 \\
\hline Medical comorbidities & $4(22.2 \%)$ & $52(23.1 \%)$ & 1.00 \\
\hline CVD & $1(5.6 \%)$ & $31(13.8 \%)$ & 0.48 \\
\hline DM & $1(5.6 \%)$ & $11(4.8 \%)$ & 1.00 \\
\hline COPD & $2(11.1 \%)$ & $11(4.8 \%)$ & 0.25 \\
\hline Smoking & $17(94.4 \%)$ & $123(54.7 \%)$ & 0.0008 \\
\hline $\begin{array}{l}\text { Prior abdominal } \\
\text { surgery }\end{array}$ & & \\
\hline
\end{tabular}

Abbreviations: BMI, body mass index; COPD, chronic obstructive pulmonary disease; CVD, cardiovascular disease; DM, diabetes mellitus; VHR, ventral hernia repair.

Table 2 Operative details

\begin{tabular}{|c|c|c|c|}
\hline & $\begin{array}{l}\text { Concurrent } \\
\text { VHR }(n=18)\end{array}$ & $\begin{array}{l}\text { No VHR } \\
(n=225)\end{array}$ & $p$-Value \\
\hline \multicolumn{4}{|l|}{ Laterality } \\
\hline Unilateral & $12(66.7 \%)$ & $140(62.2 \%)$ & \multirow[t]{2}{*}{0.80} \\
\hline Bilateral & $6(33.3 \%)$ & 85 (37.8\%) & \\
\hline \multicolumn{4}{|l|}{ Timing } \\
\hline Immediate & $10(55.6 \%)$ & $163(72.4 \%)$ & \multirow[t]{3}{*}{0.30} \\
\hline Delayed & $6(33.3 \%)$ & 44 (19.6\%) & \\
\hline Mixed & $2(11.1 \%)$ & $18(8.0 \%)$ & \\
\hline \multicolumn{4}{|l|}{ Flap type } \\
\hline MS-0 & $2(8.3 \%)$ & $20(6.5 \%)$ & \multirow[t]{4}{*}{0.24} \\
\hline MS-1 & 7 (29.2\%) & 45 (14.5\%) & \\
\hline MS-2 & $2(8.3 \%)$ & 47 (15.2\%) & \\
\hline MS-3 (DIEP) & $13(54.2 \%)$ & 198 (63.9\%) & \\
\hline $\begin{array}{l}\text { Operative } \\
\text { time (min) }\end{array}$ & $622.8 \pm 114.2$ & $598.3 \pm 100.5$ & 0.80 \\
\hline \multicolumn{4}{|l|}{ Hernia location } \\
\hline Lateral & $14(77.8 \%)$ & $\mathrm{n} / \mathrm{a}$ & \multirow[t]{2}{*}{$\mathrm{n} / \mathrm{a}$} \\
\hline Midline & $4(22.2 \%)$ & $\mathrm{n} / \mathrm{a}$ & \\
\hline \multicolumn{4}{|l|}{ Hernia repair } \\
\hline Primary & $13(72.2 \%)$ & $\mathrm{n} / \mathrm{a}$ & \multirow[t]{2}{*}{$\mathrm{n} / \mathrm{a}$} \\
\hline Mesh & $5(27.8 \%)$ & $\mathrm{n} / \mathrm{a}$ & \\
\hline
\end{tabular}

Abbreviations: DIEP, deep inferior epigastric artery perforator; MS, muscle-sparing; $n / a$, not available; VHR, ventral hernia repair.

peri-/infraumbilical, with the mean hernia size $3.0 \pm 0.9 \mathrm{~cm}$ (range, $1.5-6.0 \mathrm{~cm}$ ). No other significant differences were identified between the two groups.

Surgical details are presented in -Table 2. In the experimental group, 12 patients (66.6\%) underwent unilateral breast reconstruction and 6 patients (33.3\%) underwent bilateral breast reconstruction. Of the 24 free flaps performed, there were 13 deep inferior epigastric artery perforator flaps (54.2\%),
Table 3 Postoperative outcomes

\begin{tabular}{|l|l|l|l|}
\hline & $\begin{array}{l}\text { Concurrent } \\
\text { VHR }(\boldsymbol{n}=\mathbf{1 8})\end{array}$ & $\begin{array}{l}\text { No VHR } \\
(\boldsymbol{n}=\mathbf{2 2 5})\end{array}$ & $p$-Value \\
\hline Follow-up (mo) & $20.9 \pm 10.0$ & $15.8 \pm 4.2$ & 0.85 \\
\hline Length of stay (d) & $3.4 \pm 0.5$ & $3.1 \pm 0.4$ & 0.98 \\
\hline $\begin{array}{l}\text { Recipient-site } \\
\text { complication }\end{array}$ & $5(27.8 \%)$ & $54(24.0 \%)$ & 0.78 \\
\hline Seroma & - & $3(1.3 \%)$ & 1.00 \\
\hline Hematoma & $2(11.1 \%)$ & $11(4.9 \%)$ & 0.25 \\
\hline Dehiscence & $2(11.1 \%)$ & $18(8.0 \%)$ & 0.65 \\
\hline Infection & $1(5.6 \%)$ & $10(4.4 \%)$ & 0.58 \\
\hline Partial flap loss & - & $16(7.1 \%)$ & 0.62 \\
\hline Total flap loss & - & $6(2.7 \%)$ & 1.00 \\
\hline $\begin{array}{l}\text { Donor-site } \\
\text { complication }\end{array}$ & $5(27.8 \%)$ & $47(20.9 \%)$ & 0.55 \\
\hline Seroma & - & $4(1.8 \%)$ & 1.00 \\
\hline Hematoma & - & $6(2.7 \%)$ & 1.00 \\
\hline Dehiscence & $3(16.7 \%)$ & $12(5.3 \%)$ & 0.09 \\
\hline Infection & $2(11.1 \%)$ & $9(4.0 \%)$ & 0.19 \\
\hline Umbilical necrosis & - & $15(7 \%)$ & 0.36 \\
\hline Hernia/bulge & - & $8(3.6 \%)$ & 0.16 \\
\hline Intra-abdominal & - & - & 1.00 \\
\hline $\begin{array}{l}\text { Readmission } \\
\text { within 30 d }\end{array}$ & $1(5.6 \%)$ & $15(6.7 \%)$ & 1.00 \\
\hline $\begin{array}{l}\text { Reoperation } \\
\text { within 30 d }\end{array}$ & $2(11.1 \%)$ & $28(12.4 \%)$ & 1.00 \\
\hline
\end{tabular}

Abbreviation: VHR, ventral hernia repair.

2 muscle-sparing (MS)-2 transverse rectus abdominis myocutaneous (TRAM) flaps (8.3\%), 7 MS-1 TRAM flaps (29.2\%), and 2 MS-0 TRAM flaps (8.3\%). The mean operative time was $623 \pm 114$ minutes. There were no significant differences identified between the two groups with regard to surgical details. In the experimental group, 5 patients (27.8\%) underwent hernia repair with mesh and 13 patients (72.2\%) underwent primary repair without mesh. All hernia repairs with mesh were repaired using synthetic mesh (polypropylene) with primary fascial closure, of which two were repaired with mesh in the overlay position, and three as an underlay repair. Donor-site closure in patients without preexisting ventral hernias was performed by means of primary fascial closure using permanent suture in most cases.

Postoperative outcomes are presented in - Table 3. Overall average length of stay was $3.2 \pm 0.4$ days and the mean followup period was $20.5 \pm 5.2$ months. There was no significant difference observed in the incidence of donor-site complications in patients who underwent concurrent hernia repair and those who did not ( 27.8 vs. $20.9 \%$, respectively; $p=0.78$ ). In the experimental group, three patients (16.7\%) experienced superficial wound dehiscence of the donor site, two of which were treated with local wound care and one repaired operatively. Two patients (11.1\%) developed abdominal cellulitis and required admission for intravenous antibiotics. There were no occurrences of hernia, bulge, need for mesh explantation, or umbilical 
necrosis including in patients who had umbilical hernias. Moreover, no significant difference was present in the rates of specific types of donor-site complications between the two groups.

No significant difference was identified in the overall rates of recipient-site complications between patients who underwent concurrent hernia repair and those who did not $(27.8$ vs. $24.0 \%$, respectively; $p=0.78$ ). There were also no significant differences observed in the rates of specific types of recipient-site complications between the two groups. There were no occurrences of partial or total flap loss in the experimental group.

\section{Discussion}

The results of this study indicate that ventral hernia repair can be safely and effectively performed at the time of abdominally based microsurgical breast reconstruction. Patients with preexisting ventral hernias who underwent concurrent repair were well matched to the control group, and no differences in the rates of donor- or recipient-site complications were observed, including hernia recurrence and flap loss. The experimental group was also similar in regard to secondary outcome measures such as length of stay, operative time, and rates of readmission and reoperation.

The successful harvest and transfer of all free flaps in the experimental group may have been due, in part, to the fact that the dissection involved is fundamentally not different from the dissection that microsurgeons routinely perform in patients who have had prior abdominal surgery, where there can often be extensive scarring to perforators and the pedicle. Adherence to established principles of retrograde perforator dissection, atraumatic technique, and preservation of salvage options appears to allow for safe flap dissection in patients with preexisting ventral hernias. In addition, we believe that preferential selection of perforators uninvolved with the hernia (i.e., use of lateral row perforators when hernias are midline, and medial row perforators when hernias are lateral), when possible, can facilitate flap harvest in these cases.

The absence of hernia recurrence in the experimental group and similarity in the rates of donor-site complications compared with the control group suggest that the incisions and exposure required in abdominally based breast reconstruction do not compromise the integrity of the hernia repair. Although a lower transverse incision may not always be the approach of choice in a patient undergoing ventral hernia repair, the wide abdominal exposure that occurs in the course of flap harvest nevertheless provides ready access to the hernia defect. In our experience, we feel that it may be advisable to repair the hernia only after completing flap elevation, which allows the hernia repair to take into account any effects of harvest itself, such as any fascial or muscle resection performed and alterations in the tension of the fascial closure. A fascial-sparing approach to fasciotomies may be advisable in these cases in regard to minimizing tension on the repair and facilitating suture fixation of the mesh. Newer techniques such as robotic-assisted flap harvest may be useful to minimize anterior rectus sheath dissection in the interest of optimizing hernia repair. ${ }^{10}$
Although the subject of the outcomes of abdominally based breast reconstruction in patients with preexisting ventral hernias has been relatively unaddressed in the literature, there has been extensive research published on breast reconstruction patients with a history of prior abdominal surgery. ${ }^{11}$ These studies primarily focus on optimizing flap perfusion in relation to preexisting abdominal scars. ${ }^{12}$ Much of this work finds that common abdominal scars, such as Pfannenstiel, midline, or laparoscopy incisions, do not appear to impact complication rates, and that abdominally based breast reconstruction can be safely performed in this setting. ${ }^{11,13}$ Some authors have reported an increase in donor-site complications when there are preexisting abdominal scars, but that these problems can be mitigated by modifying the design of the flap. ${ }^{13,14}$ For example, when subcostal incisions are present, complications that can occur due to alterations in perfusion to the abdominal wall can be minimized through the use of perforator-sparing techniques in the upper abdomen. ${ }^{15,16}$

Several studies have examined the safety of concurrent ventral hernia repair in the setting of panniculectomy. ${ }^{17,18}$ Overall, this research indicates that concurrent ventral hernia repair and panniculectomy appears to be associated with an increased risk of complications, especially wound dehiscence and surgical site infection, and thus it may be prudent in these cases to consider staging of the procedures. ${ }^{19-21}$ Although this procedure has similar elements to concurrent ventral hernia repair and abdominally based breast reconstruction, one notable difference lies in the characteristics of the patient populations involved. Patients undergoing panniculectomy generally have a higher body mass index and greater medical comorbidities, which may help explain the differences in the findings of this study compared with prior studies of panniculectomy patients. ${ }^{19}$

This study has several limitations. First, due to its retrospective design, not all variables that may have had an impact on the study outcome measures were available (e.g., flap and hernia dimensions) or controlled for (e.g., reconstructive surgeon). In addition, the relatively small sample size of patients who underwent concurrent hernia repair limits the degree to which conclusions may be drawn and their generalizability. A multicenter study would most likely be best suited to further investigate this subject with sufficient power. Finally, there is the possibility of selection bias, as there may have been patients with preexisting ventral hernias that were evaluated, but, for a variety of potential reasons, did not undergo abdominally based breast reconstruction (e.g., extensive hernia and deemed not a suitable candidate for abdominally based breast reconstruction). Further, larger and prospective studies are needed to further define the relationship between concurrent ventral hernia repair and abdominally based microsurgical breast reconstruction.

\section{Conclusion}

In patients undergoing abdominally based microsurgical breast reconstruction, concurrent ventral hernia repair in patients with preexisting hernias is associated with comparable outcomes to that of patients without hernias. 
Funding

None.

\section{Conflict of Interest \\ None declared.}

\section{References}

1 Granzow JW, Levine JL, Chiu ES, Allen RJ. Breast reconstruction with the deep inferior epigastric perforator flap: history and an update on current technique.J Plast Reconstr Aesthet Surg 2006;59(06):571-579

2 Holoyda KA, Simpson AM, Ye X, Agarwal JP, Kwok AC. Immediate bilateral breast reconstruction using abdominally based flaps: an analysis of the nationwide inpatient sample database. J Reconstr Microsurg 2019;35(08):594-601

3 Kwok AC, Simpson AM, Ye X, Tatro E, Agarwal JP. Immediate unilateral breast reconstruction using abdominally based flaps: analysis of 3,310 cases. J Reconstr Microsurg 2019;35(01):74-82

4 Center for Disease Control United States Cancer Statistics Data Visualizations. Available at: https://gis.cdc.gov/grasp/USCS/DataViz.html. Accessed December 8, 2019

5 Roostaeian J, Yoon AP, Sanchez IS, et al. The effect of prior abdominal surgery on abdominally based free flaps in breast reconstruction. Plast Reconstr Surg 2014;133(03):247e-255e

6 Vyas RM, Dickinson BP, Fastekjian JH, Watson JP, Dalio AL, Crisera CA. Risk factors for abdominal donor-site morbidity in free flap breast reconstruction. Plast Reconstr Surg 2008;121(05):1519-1526

7 Mehrara BJ, Santoro TD, Arcilla E, Watson JP, Shaw WW, Da Lio AL. Complications after microvascular breast reconstruction: experience with 1195 flaps. Plast Reconstr Surg 2006;118(05):1100-1109 , discussion 1110-1111

8 Laporta R, Longo B, Sorotos M, Santanelli di Pompeo F. Tips and tricks for DIEP flap breast reconstruction in patients with previous abdominal scar. Microsurgery 2017;37(04):282-292

9 Doval AF, Lamelas AM, Daly LT, et al. Deep inferior epigastric artery perforator flap breast reconstruction in women with previous abdominal incisions: a comparison of complication rates. Ann Plast Surg 2018;81(05):560-564
10 Manrique OJ, Bustos SS, Mohan AT, et al. Robotic-assisted DIEP flap harvest for autologous breast reconstruction: a comparative feasibility study on a cadaveric model. J Reconstr Microsurg 2020

11 Di Candia M, Asfoor AA, Jessop ZM, Kumiponjera D, Hsieh F, Malata CM. Previous multiple abdominal surgeries: a valid contraindication to abdominal free flap breast reconstruction? Eplasty 2012;12:e31

12 Hsieh F, Kumiponjera D, Malata CM. An algorithmic approach to abdominal flap breast reconstruction in patients with pre-existing scars-results from a single surgeon's experience. J Plast Reconstr Aesthet Surg 2009;62(12):1650-1660

13 Dayhim F, Wilkins EG. The impact of Pfannenstiel scars on TRAM flap complications. Ann Plast Surg 2004;53(05):432-435

14 Parrett BM, Caterson SA, Tobias AM, Lee BT. DIEP flaps in women with abdominal scars: are complication rates affected? Plast Reconstr Surg 2008;121(05):1527-1531

15 Takeishi M, Shaw WW, Ahn CY, Borud LJ. TRAM flaps in patients with abdominal scars. Plast Reconstr Surg 1997;99(03):713-722

16 Schoeller T, Huemer GM, Kolehmainen M, Otto-Schoeller A, Wechselberger G. Management of subcostal scars during DIEPflap raising. Br J Plast Surg 2004;57(06):511-514

17 Hughes KC, Weider L, Fischer J, et al. Ventral hernia repair with simultaneous panniculectomy. Am Surg 1996;62(08):678-681

18 Saxe A, Schwartz S, Gallardo L, Yassa E, Alghanem A. Simultaneous panniculectomy and ventral hernia repair following weight reduction after gastric bypass surgery: is it safe? Obes Surg 2008;18 (02):192-195, discussion 196

19 Fischer JP, Tuggle CT, Wes AM, Kovach SJ. Concurrent panniculectomy with open ventral hernia repair has added risk versus ventral hernia repair: an analysis of the ACS-NSQIP database. J Plast Reconstr Aesthet Surg 2014;67(05):693-701

20 Fischer JP, Basta MN, Wink JD, Wes AM, Kovach SJ. Optimizing patient selection in ventral hernia repair with concurrent panniculectomy: an analysis of 1974 patients from the ACS-NSQIP datasets. J Plast Reconstr Aesthet Surg 2014;67(11):1532-1540

21 Warren JA, Epps M, Debrux C, et al. Surgical site occurrences of simultaneous panniculectomy and incisional hernia repair. Am Surg 2015;81(08):764-769 